\title{
A Life Course Health Development Perspective on Oral Health
}

\author{
James J. Crall and Christopher B. Forrest
}

\section{Introduction}

\subsection{Life Course Health Development Concepts and Principles}

Life Course Health Development(LCHD) is a conceptual framework that helps to explain how health develops over an individual's lifetime and emphasizes the need to treat health development as a long-term investment, beginning early and continuing throughout life (Halfon and Hochstein 2002). LCHD provides a powerful approach to understanding diseases and conditions and how risk factors, protective factors, critical life experiences, and environments affect long-term health and disease outcomes. LCHD also can help examine and explain how health and disease patterns, particularly health disparities, develop across populations and over time (Halfon and Hochstein 2002; Ben-Shlomo and Kuh 2002; Keating and Hertzman 1999).

\section{J.J. Crall ( $\bowtie)$}

Division of Public Health and Community Dentistry, University of California Los Angeles (UCLA) School of Dentistry, Los Angeles, CA, USA e-mail: jcrall@dentistry.ucla.edu

\section{C.B. Forrest}

Applied Clinical Research Center, Children's

Hospital of Pennsylvania, Philadelphia, PA, USA
Efforts to relate the life course perspective or life course theory to the field of maternal and child health (Fine and Kotelchuck, 2010) have emphasized the following key concepts:

Pathways or Trajectories Health pathways or trajectories are constructed and modified throughout the life span. While individual trajectories vary, general patterns can be predicted for populations and communities based on social, economic, and environmental exposures and experiences. A life course does not reflect a series of discrete steps or stages, but rather an integrated, dynamic, and continuous set of exposures and experiences. The set of possible trajectories that a person can experience is constrained by evolutionary forces and is highly determined by exposure to various environmental contexts.

Importance of Early Life Exposures Early experiences can markedly influence an individual's future health development. Of particular salience are exposures that occur prenatally (i.e., exposures in utero) and intergenerationally (i.e., factors related to the health of the mother prior to conception). While adverse events and exposures can have an impact at any point in a person's life course, the impact may be greatest at specific critical or sensitive periods when developing biological systems are most readily modified (e.g., during fetal development, in early childhood, during adolescence). 
Cumulative Impact Cumulative experiences also can influence an individual's future health and development, even though any individual experience may not impact health development. For example, individuals may adapt with minimal impact to each episode of stress; however, the cumulative impact of multiple stresses over time (also referred to as "weathering" or "allostatic load") may have a profound direct impact on health development by altering biological function and human behavior.

Risk and Protective Factors Throughout the life span, protective factors improve health and contribute to health development, while risk factors diminish health and make it more difficult to reach full health potential. Moreover, pathways are changeable, and risk and protective factors are not limited to individual behavioral patterns or receipt of health-care and social services, but also include factors related to family, neighborhood, community, and social policy.

The LCHD theoretical framework (presented more thoroughly in Chap. 2) incorporates and expands these concepts into a robust explanatory synthesis of how health in individuals and populations is produced and modified. In this chapter, we use the LCHD principles to guide our review of oral health development. The Table 1 illustrates each LCHD principle's relevance to oral health.

\subsection{Previous Efforts to Apply Life Course Concepts to Oral Health}

The multifactorial etiology of oral conditions, their chronic nature and occurrence over the life span, and inherent features of their expression make oral health well suited to studies which seek to apply life course concepts. For example, Nicolau and colleagues (Nicolau et al. 2007) have highlighted several features that make oral conditions amenable to life course epidemiological studies, the first of which is their observability -i.e., once these conditions develop, they are readily detectable during assessments as opposed to conditions that resolve or go unnoted if study participants fail to report them. A second feature relates to their being cumulative conditions, which allows for comparisons of the degree of disease development among individuals so that, rather than all who develop the disease being enumerated together, distinctions can be made among them with respect to the extent or severity of their disease. Third, oral conditions can be reliably measured and validly diagnosed without sophisticated or costly technology. Fourth, these conditions are moderately prevalent; thus, the required sample size for cohort studies is manageable. And, finally, oral health conditions have a public health importance that makes their study justifiable on both ethical and economic grounds.

Despite growing interest in using life course concepts as the basis for various oral healthrelated scientific studies, robust applications have been rather limited. A PubMed search conducted as part of the literature review for this chapter using the terms "life course and oral health" yielded over 500 citations. However, a substantial portion of the publications examined in this and other literature searches were found to have one or more of the following limitations: studies focused on a single condition, used crosssectional data, or analyzed a relatively narrow portion of the life span, studies were based on relatively small sample sizes, and studies were predicated on retrospective analyses that frequently relied on recall of rather distant events.

Furthermore, efforts to examine oral health from the perspective of LCHD concepts and principles have not been pursued. Therefore, the goals of this chapter are to (a) examine the concept of oral health and major oral diseases and conditions from a LCHD perspective using the key concepts and principles noted in the table, (b) assess what is (and what is not) known about the development of oral health and its impact on general health and well-being across the life course, and (c) offer recommendations for future research. In light of the evidence-based literature shortcomings noted in the preceding paragraph, assessments of what is known about applications of life course research approaches to studies of oral health are limited to research publications consistent with the life course epidemiology 


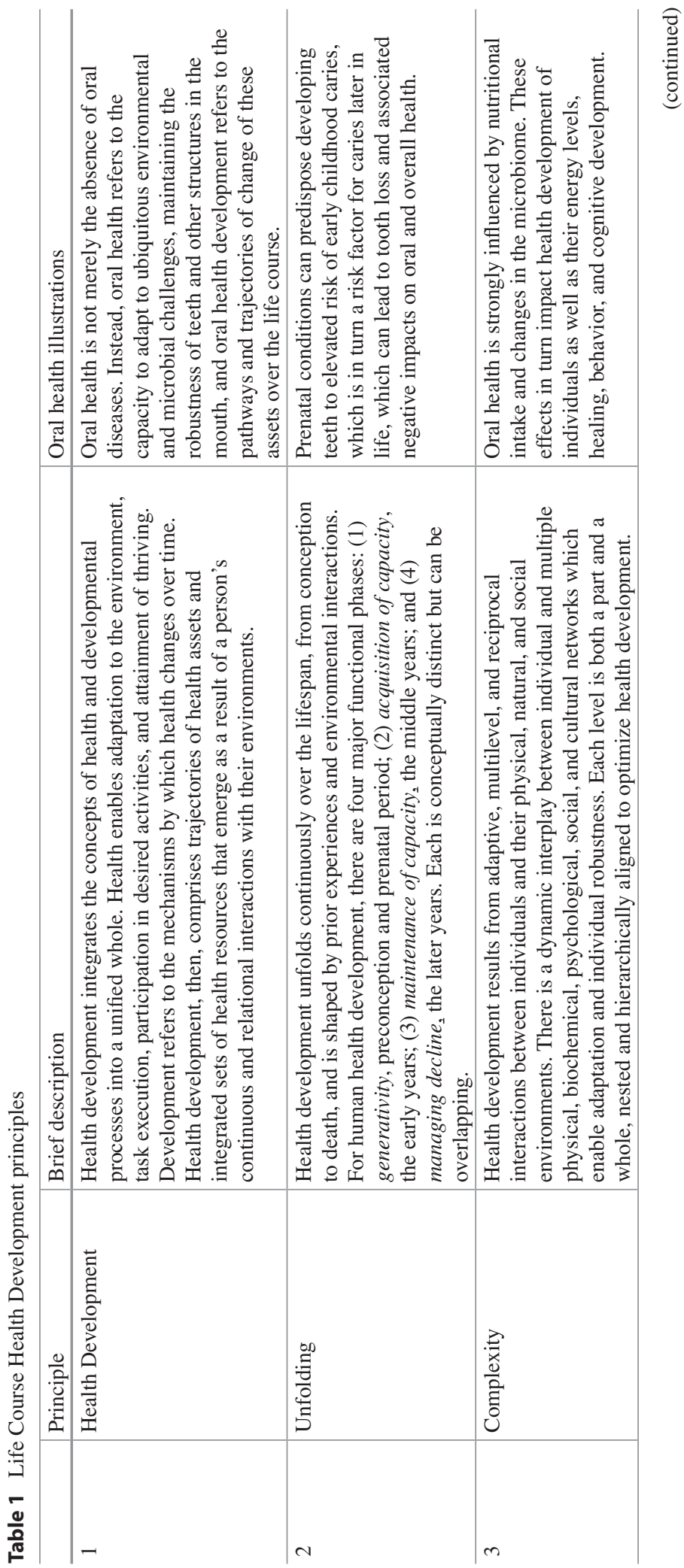




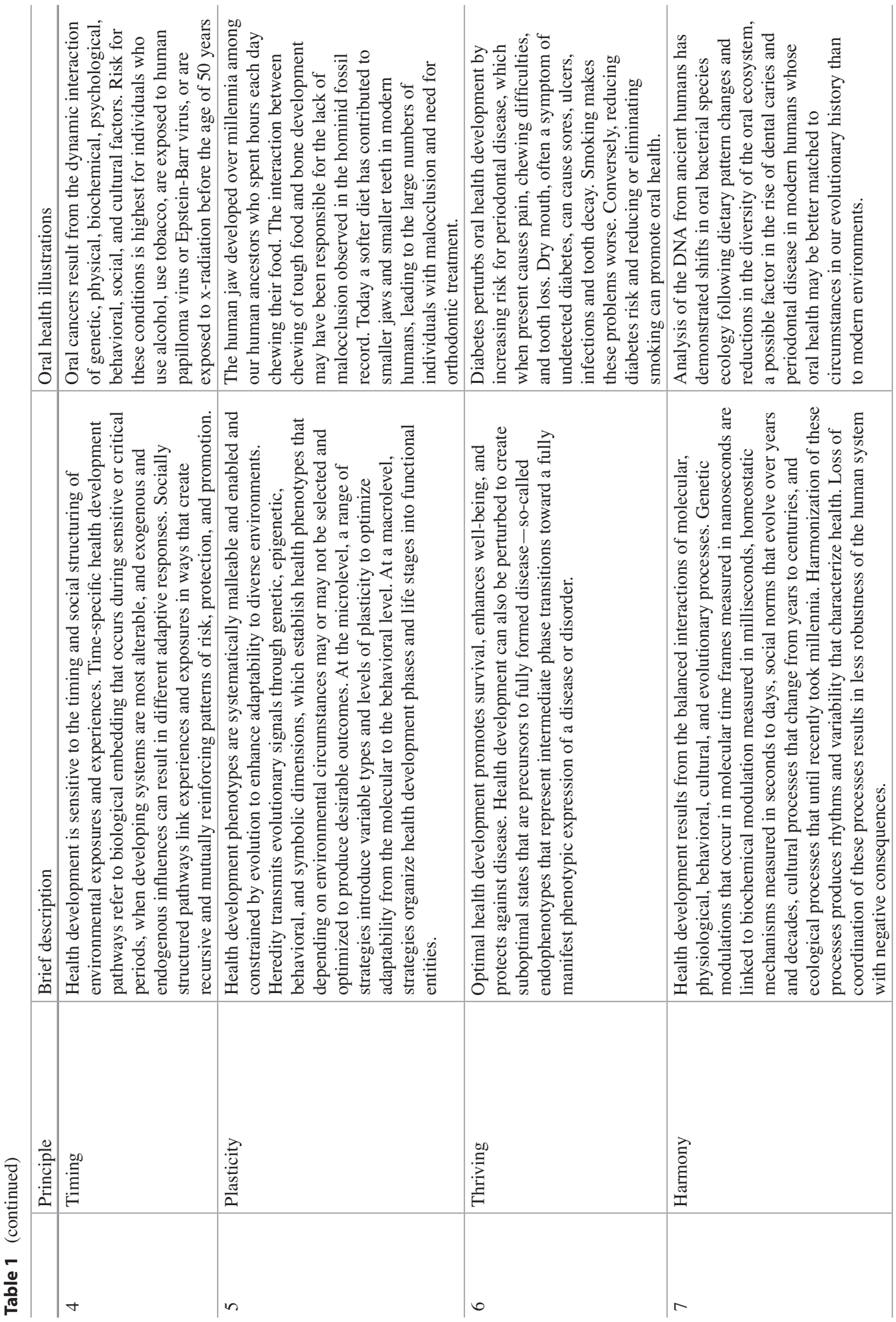


standard and LCHD framework. Furthermore, because of the diverse nature of the major oral health-related conditions noted above, assessments are largely limited to "dental conditions" (i.e., dental caries, periodontal diseases, and tooth loss), with limited additional examples pertaining to craniofacial abnormalities, oral cancers, or temporomandibular joint dysfunction.

\section{Conceptualizing Oral Health and Overview of Major Conditions}

\subsection{Conceptualizing Oral Health}

Concepts and definitions of oral health have varied over time, often paralleling broader conceptualizations of health. Concepts of how oral health changes over time also often parallel more general paradigms. For example, many recent concepts of oral health have been based on the biopsychosocial model, which focuses on how the integration of various aspects of biological, psychological, and social domains influence individuals' health in different contexts and over time (Borrell-Carrio et al. 2004).

The US Surgeon General's Report on Oral Health (SGROH), issued in 2000, and ensuing initiatives have emphasized two major themes regarding the concept of oral health-i.e., oral health means much more than healthy teeth, and oral health is integral to general health (US Department of Health and Human Services 2000). Interestingly, the SGROH embraced the World Health Organization (WHO) definition of oral health as "a state of being free from mouth and facial pain, oral and throat cancer, oral infection and sores, periodontal (gum) disease, tooth decay, tooth loss, and other diseases and disorders that limit an individual's capacity in biting, chewing, smiling, speaking, and psychosocial wellbeing." This definition espoused by both the WHO and SGROH is deficient in that it does not positively define what oral health is, but rather presents the concept as the absence of detrimental conditions or disorders. Conflating oral health and oral disorders is contrary to the WHO definition of overall health which states that health is more than just the absence of disease and represents a state of complete physical, mental, and social well-being. Researchers working to develop measures related to oral health and its impact on quality of life also have concluded that health and disease are different domains of human experience, which vary both over time and among individuals, and are dependent on context (Locker and Slade 1994; Gregory et al. 2005).

A recent development in the conceptualization and definition of oral health has emerged from the FDI World Dental Federation in late 2016. According to the new FDI definition, "Oral health is multifaceted and includes the ability to speak, smile, smell, taste, touch, chew, swallow, and convey a range of emotions through facial expressions with confidence and without pain, discomfort, and disease of the craniofacial complex. The core elements of oral health in the FDI framework are as follows: disease and condition status refers to a threshold of severity or a level of progression of disease, which also includes pain and discomfort; physiological function refers to the capacity to perform a set of actions that include, but are not limited to, the ability to speak, smile, chew, and swallow; and psychosocial function refers to the relationship between oral health and mental state that includes, but is not limited to, the capacity to speak, smile, and interact in social and work situations without feeling uncomfortable or embarrassed." (Glick et al. 2016).

LCHD theoretical principles conceptualize health as an emergent set of integrated assets that enable adaptation and pursuit of meaning and happiness, reflect broader notions of the impact of various health determinants over time, and consider health in a more positive context (see Table 1). Applying LCHD concepts, individuals with disease (e.g., diabetics or persons living with AIDS) can also be considered healthy if they have developed assets which enable adaptation to their environment, task execution, participation in desired activities, and an ability to thrive; health and disease are distinct, albeit interrelated concepts.

LCHD builds and expands upon the basic tenets of the biopsychosocial and other ecologi- 
cal models and establishes a conceptual foundation for a future era of health care that moves beyond systems focused on management of acute and chronic diseases and conditions to systems whose focus is on optimizing lifelong health for individuals and populations (Halfon et al. 2014). Using LCHD concepts and principles to conceptualize and define oral health has the potential to move practitioners, researchers, policy makers, and the public beyond their historical limited focus on the physical form and function of structures in and surrounding the oral cavity and the clinical consequences of oral/dental diseases to a new paradigm - one which fully embraces LCHD tenets and the development of more robust LCHD-based policies and systems for optimizing the oral health of individuals and populations.

\subsection{Major Oral Health-Related Diseases and Conditions}

Clinical conditions that have major significance with respect to oral health in terms of prevalence, impact on health, development and well-being, and economic considerations include dental diseases and their consequences (dental caries, periodontal diseases, tooth loss), craniofacial developmental disorders (e.g., cleft lip, cleft palate, malocclusion), oral cancers (including pharyngeal and salivary gland cancers), and facial pain (distinct from dental pain) generally associated with temporomandibular joint and muscular disorders. Brief overviews of these conditions are provided below.

Dental Conditions Despite significant progress during the latter portion of the twentieth century, dental caries (tooth decay) remains the most common chronic disease of childhood and a major cause of tooth loss in children and adults. In the United States, approximately $25 \%$ of 2-5-year-olds and over 50\% of 6-8-year-old children experience dental caries in their primary teeth, and over $50 \%$ of $12-15$-year-olds and $67 \%$ of 16-19-year-olds experience caries in their permanent teeth (Dye et al. 2011a). Although substantial declines in the average number of teeth and tooth surfaces affected by caries have been documented in recent decades, particularly in developed countries, according to the WHO, the prevalence of tooth decay in school-age children globally ranges from $60 \%$ to $90 \%$ (WHO, The World Oral Health Report 2008).

Significant disparities in childhood caries experience also persist. On average, US children from lower-income households and children of color are three to five times more likely than their white more affluent counterparts to experience caries and exhibit more severe forms of tooth decay (Dye et al. 2011a; Vargas et al. 1998). Of interest from a LCHD perspective, many children from high-risk population groups do not exhibit severe forms of dental caries, presumably because the balance between individual's risk and protective factors (e.g., diet, toothbrushing habits, fluoride exposure, oral microflora or other unidentified characteristics) is conducive to maintaining a healthy dentition (Feathersone 2000).

Caries affects dentate individuals across the life span (Fig. 1), with over $90 \%$ of US adults with natural teeth experiencing dental caries in their permanent teeth (Dye et al. 2011a, b). According to the WHO (WHO, The World Oral Health Report, 2008) nearly $100 \%$ of dentate adults globally are affected by dental caries, making it the most prevalent chronic condition of people worldwide. From a life course perspective, early childhood caries can have a profound deleterious lifelong effect on an individual's dentition status, as ECC often is a precursor of caries and its consequences in adults.

Periodontal disease mainly results from infections and inflammation of the gingiva (gums) and bone that surround and support the teeth. In its early stage, called gingivitis, the gums can become swollen and red and may bleed. In its more serious form, called periodontitis, the gums can pull away from the teeth, bone can be lost, and teeth may loosen or even fall out. Thirty percent of US adults $30+$ years of age have moderate periodontal disease, and $8.5 \%$ have severe periodontitis (Thornton-Evans 2013). Males, older adults, Black and Hispanic adults, current smokers, and those with lower incomes and less education are more likely to have moderate or severe 


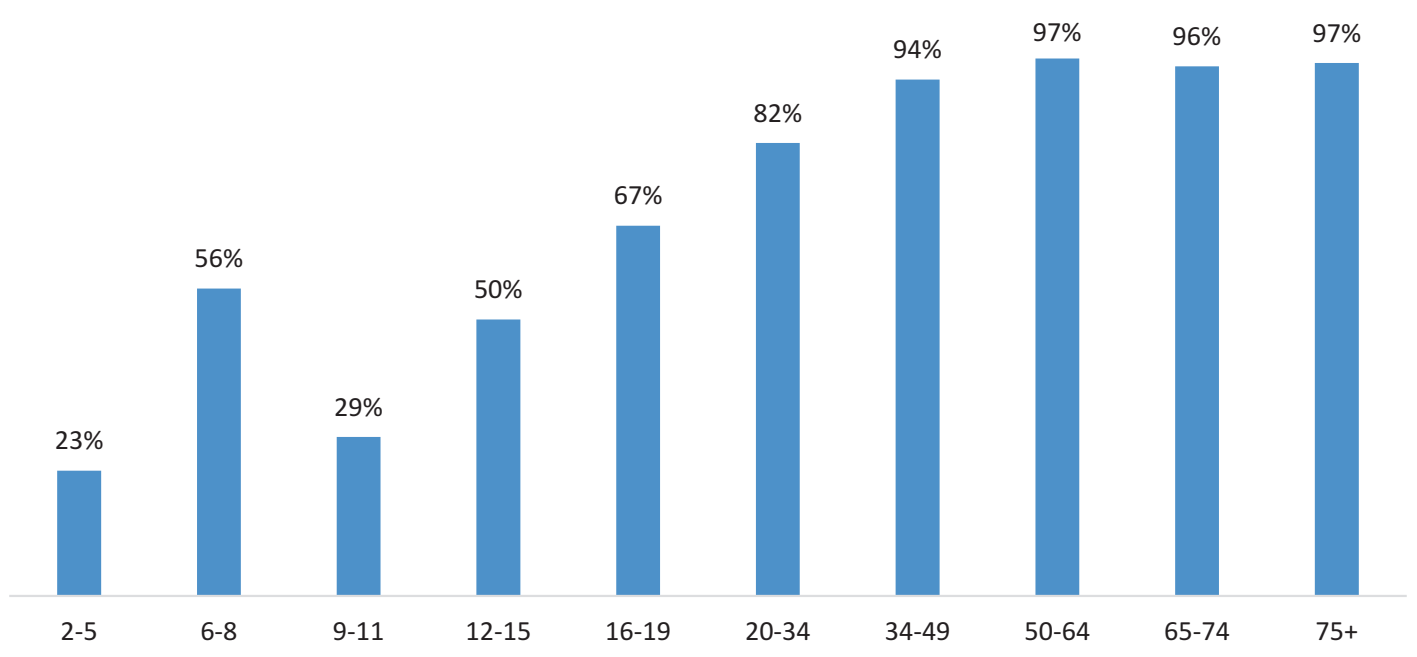

Fig. 1 Proportion of the US population with caries experience (percentages for 2-5- and 6-8-year-olds reflect the percentage of individuals with caries experience in primary teeth; for ages 9 and above, percentages reflect the

percentage with caries experience in permanent teeth) by age (years) (Data Source: National Health and Nutrition Examination Survey, 2011-2012. National Center for Health Statistics, CDC)

periodontal disease (Thornton-Evans 2013). Globally, severe periodontal disease is found in $15-20 \%$ of adults aged 35-44 years and increases in prevalence with advancing age (WHO, The World Oral Health Report 2008).

Dental caries and periodontal disease are the primary reasons for tooth loss (Dye et al. 2011b; Thornton-Evans 2013) which in its more severe forms (loss of multiple teeth) can have negative psychological, social, nutritional, and physical effects. A person's quality of life is diminished as a result of tooth loss due to reductions in their ability to chew and speak and reduced social interactions and self-esteem (US Department of Health and Human Services 2000; Hollister \& Weintraub 1993; Brennan et al. 2008). National survey data indicate that approximately $50 \%$ of US adults have lost at least one tooth. Approximately 5\% of US adults overall and 25\% of adults aged 65+ are completely edentulous (have no natural teeth) (Dye et al. 2011b). Worldwide, about $30 \%$ of people aged $65-74$ have no natural teeth, with considerable disparities across countries (WHO, The World Oral Health Report 2008).

Total US spending for dental services has been relatively flat at approximately $\$ 111$ billion since 2010 (Wall 2013). The bulk of dental care spending is related to diagnosis, prevention and treatment of dental caries and periodontal disease, and treatment related to removal and replacement of lost teeth.

Craniofacial Conditions Craniofacial defects such as cleft lip and cleft palate (CLP) are among the most common of all birth defects. The US incidence of cleft palate is 6 per 10,000 live births, and the incidence for cleft lip with or without cleft palate is 11 per 10,000 live births (Center for Disease Control and Prevention (CDC) 2014). CLP can occur as an isolated condition or may be a component of an inherited disease or syndrome. Cleft lip and cleft palate are thought to be caused by a combination of genes and other factors, such as things the mother comes in contact with in her environment, what the mother eats or drinks, or certain medications she uses during pregnancy. Maternal smoking, alcohol use, steroid use, and anticonvulsants are associated with increased risk for cleft lip and palate (Kohli and Kohli 2012). The incidence of CLP also varies by race, with Asians and Native Americans having higher rates and AfricanAmericans having lower rates. According to the Centers for Disease Control and Prevention 
(CDC), in the United States, cleft lip and palate is the third most common birth defect, and health expenditures are approximately eight times higher in the first 10 years of life for children with clefting than for those without (Boulet et al. 2009).

Malocclusion Misalignment or abnormal positioning of the teeth or an incorrect relation between the teeth in the upper and lower jaws - is neither a disease nor a life-threatening condition. Nevertheless, growing numbers of people seek and undergo orthodontic treatment, often because of esthetic concerns and other quality of life issues (Liu et al. 2009). Although children make up the majority of orthodontic patients, adults increasingly are seeking treatment for malocclusion and now comprise one-fifth of all orthodontic patients in the US. Orthodontic services account for approximately one-eighth of US dental care expenditures (Agency for Healthcare Research and Quality 2015).

Oral Cancers Approximately 45,000 Americans and over 450,000 people worldwide are diagnosed each year with cancers that affect the mouth and/or pharynx. Oral cancers comprise $85 \%$ of all head and neck cancers and have relatively high mortality rates (5-year survival $=63 \%$ ) (The Oral Cancer Foundation 2015; American
Cancer Society 2015). Oral cancer occurrence rates are significantly higher for males than for females (except in American Indians/Alaska Natives) and higher for black males than for white males up to age 70 . Oral cancer rates increase with age, increase more rapidly after age 50, and peak between ages 70 and 80 (Fig. 2) (Ram et al. 2011). Extensive case-control and longitudinal studies have implicated tobacco and alcohol as major risk factors for oral cancer. Human papillomavirus, syphilis, oro-dental factors, dietary deficiencies, chronic candidiasis, and viruses also have been shown to be significantly associated with oral cancer (Ram et al. 2011). Findings of a recent study (Jacobson et al. 2012) suggest that oral cancers may be among the most costly forms of cancer to treat in the United States.

Facial Pain Associated with Temporoman dibular Joint Disorders The most common cause of facial pain is a group of conditions called temporomandibular joint and muscle disorders (TMJD). These disorders cause recurrent or chronic pain and dysfunction in the jaw joint and its associated muscles and supporting tissues. TMJD are the second most commonly occurring musculoskeletal condition resulting in pain and disability (after chronic low back pain), affecting

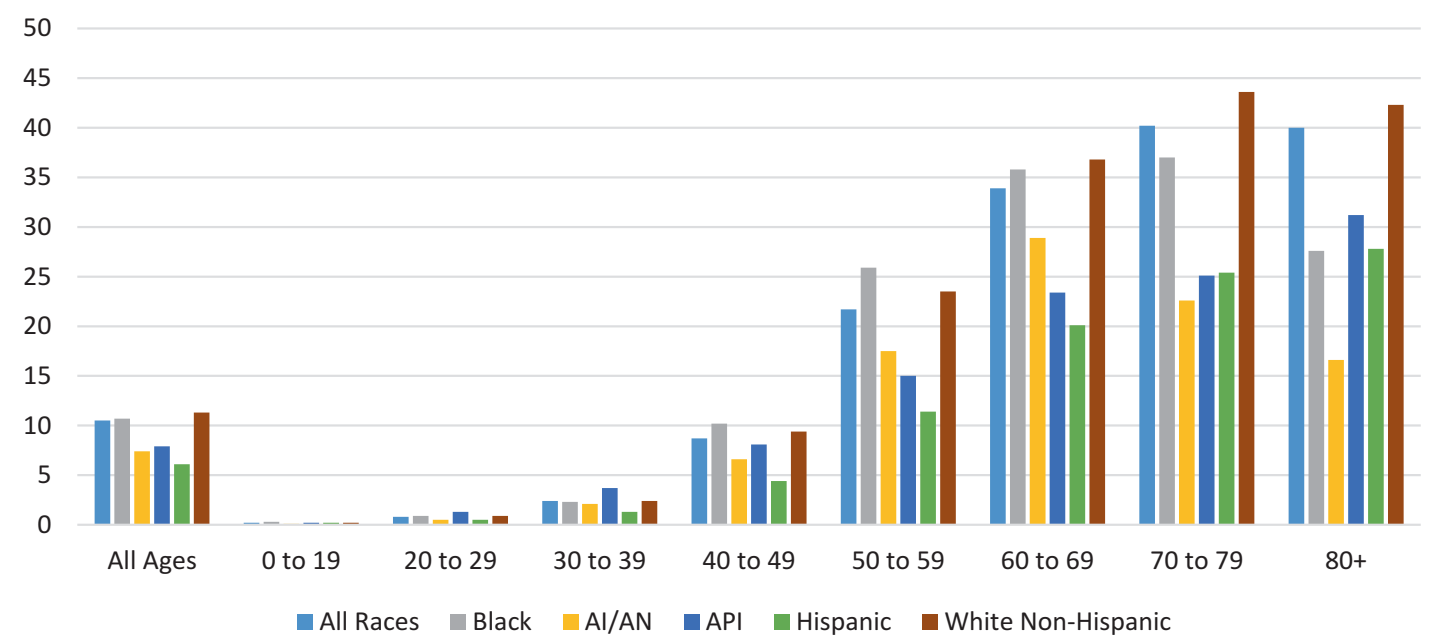

Fig. 2 Incidence of oral cancer cases per 100,000 among different age and racial groups (Data Source: Oral Cancer Incidence by Age, Race and Gender, 2009. National Institute of Dental and Craniofacial Research) 
approximately $5-12 \%$ of the population, with an annual cost estimated at $\$ 4$ billion. About half to two-thirds of those with TMJD seek treatment; among those seeking treatment, approximately 15\% develop chronic TMJD (NIDCR 2003).

\section{$3 \quad$ Evolution of Oral Health Paradigms}

The evolution of concepts regarding oral health and associated analytical models designed to measure oral health in the aggregate and its various components have paralleled the evolution of general health concepts, albeit frequently with a variable time lag. Ancient theories and hypotheses have given way to more scientifically grounded concepts reflecting changing scientific paradigms, initially based on biomedical models of disease causation, followed by biopsychosocial models that attempted to incorporate a wider range of factors influencing health and, more recently, Life Course Health Development. The following section provides a broad overview of that progression, focusing primarily on concepts regarding dental conditions.

\subsection{Changing Concepts of Dental Disease: From Worms to Germs to Chronic Disease Terms}

Tooth Worm Theory The concept of a tooth worm which according to prevailing popular beliefs caused caries and periodontitis existed in diverse cultures across the ages and, despite being labeled by medical doctors as superstition during the time of the Enlightenment, persisted in some cultures into the twentieth century. Numerous popular "therapies" were applied to eradicate the tooth worm, including fumigations with henbane seeds, magical formulas, and oaths (Gerabek 1999).

\section{Chemo-Parasitic and Focal Infection} Theories In 1890, W.D. Miller (an American dentist and the first oral microbiologist), building on Pasteur's discovery that bacteria can ferment sugars into lactic acid, formulated the chemoparasitic theory of caries. This theory held that tooth decay is caused by acids, produced by oral bacteria following fermentation of ingested sugars, which lead to loss of mineral from teeth (demineralization) (Miller 1890). Miller's second major contribution was the focal infection theory, which hypothesized that oral microorganisms or their products have a role in the development of a variety of diseases in sites removed from the oral cavity, including brain abscesses, pulmonary diseases, and gastric problems (Miller 1891). Later work by Keyes in the 1960s led to explanations of the mechanisms by which dental caries develops based on the interaction of cariogenic bacteria in biofilm/dental plaque and dietary substrates that lead to acid production, which in turn leads to demineralization of tooth structure (illustrated in the Venn diagram in Fig. 5).

Concept of Caries as a Specific and Transmissible Infection The concept of dental caries being infectious and transmissible is based on the biomedical model of disease causation, and grew out of well-designed rodent studies performed by Keyes (Keyes 1960) showing that caries only developed in rodents when they were caged with or ate the fecal pellets of groups of caries-active rodents. Further proof emerged when certain streptococci isolated from caries lesions in hamsters, unlike other types of streptococci, caused rampant decay in previously cariesinactive animals (Fitzgerald and Keyes 1961). The bacteria, later identified as Streptococcus mutans (SM), gave rise to the concept of caries being due to a specific infection with mutans streptococci (MS), a concept that has gained wide support within the field of caries microbiology (Fejerskov 2004). Subsequent studies conducted from the mid-1970s through the 1990s demonstrated that infants acquire MS from their mothers and that MS can colonize the mouths of infants even before teeth erupt (Berkowitz 2006).

Conceptualizing Caries as a Complex, Chronic Disease More recently, Featherstone (Feathersone 2000) used a modification of the chronic disease 
model, referred to as the "caries balance," to describe caries as a dynamic process that depends on the balance between constellations of risk factors (e.g., high levels of cariogenic bacteria, reduced salivary function, cariogenic dietary practices, tooth structure anomalies) and protective factors (e.g., salivary components and flow, exposure to fluorides, anti-cariogenic dietary components). Although the caries balance concept highlights the importance of risk factors and protective factors and the potential for dynamic changes over time, published examples generally depict factors related to biomedical models rather than broader biopsychosocial models.

The shift from conceptualizing dental caries based on biomedical models to models based on biopsychosocial theory represents a relatively recent paradigm change. These newer models recognize that caries expression not only depends on the complex interplay between saliva, dietary habits, and many biological determinants related to biofilm composition and metabolism; it also depends on those factors acting in concert with innumerable other biological, behavioral, and social factors acting at the level of individuals and populations. In summarizing the implications of this paradigm shift, Fejerskov (Fejerskov 2004) highlighted the following:

\footnotetext{
By appreciating that dental caries belongs to the group of common diseases considered as 'complex' or 'mulifactorial' such as cancer, heart diseases, diabetes, and certain psychiatric illnesses, we have to realize that there is no simple causation pathway. It is not a simplistic problem such as 'elimination of one type of microorganism', or a matter of improving 'tooth resistance'. Complex diseases cannot be ascribed to mutations in a single gene or to a single environmental factor. Rather
}

they arise from the concerted action of many genes, environmental factors, and risk-conferring behaviors. ... [This concept also] explains why dental caries has to be controlled lifelong if a functional dentition is to be maintained.

These new concepts explain why ... several of the 'old' recommended preventive programs are no longer effective. It is of course not because the agents we used in prevention are no longer efficacious. They just become ineffective because the caries incidence rate has changed as the environment has changed.

This brief overview highlights changes in popular and scientific concepts regarding the etiology of common dental diseases and methods for preventing or minimizing their consequences over time. Nevertheless, the persistence of relatively high levels of dental disease in sizeable segments of the population and substantial disparities within and across populations serve to highlight the limitations of traditional concepts and approaches.

\subsection{Common Risk Factors, Social Determinants, and Ecological Models}

Common Risk Factors As noted above, there is growing recognition that major oral healthrelated conditions are complex (multifactorial), chronic conditions whose occurrence and severity across the lifespan depend on interactions of a broad array of biological, behavioral, social, and environmental factors, which also are implicated in other chronic diseases and conditions as indicated in Fig. 3 (Fejerskov 2004; Sheiham and
Fig. 3 Common risk factor model of oral disease and other chronic diseases (Source: Tomar 2012)

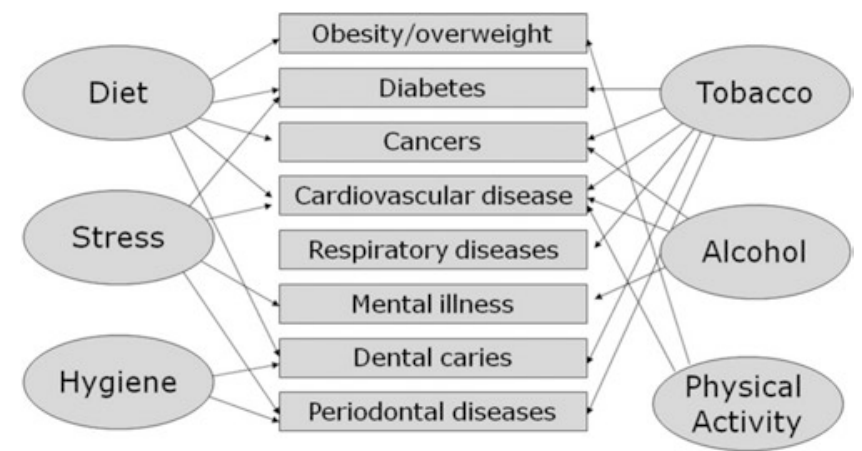


Watt 2000; Larson et al. 2008; Tomar 2012). We now realize that different types of organisms are involved in the etiology of dental caries and periodontal diseases and that the ecology of the oral microbiome is influenced by an array of common biological and biopsychosocial factors. We recognize that craniofacial disorders are caused by developmental disturbances that occur in utero and are influenced by genetic and environmental factors. We know that oral cancers result from mutations in genes that control cell behavior and are influenced by exposure to carcinogens (most notably tobacco) and biological factors, including viruses and fungi. Evidence also indicates that risk factors implicated in TMJD include poor alignment of teeth, stress, parafunctional habits, arthritis, trauma, and structural developmental abnormalities.

Cumulative Impact of Multiple Social Determinants Larson et al. (Larson et al. 2008) highlighted the cumulative impact of multiple social risk factors (limited parental education, low family income, single-parent household, race, being uninsured, family conflict, poor maternal mental health, living in an unsafe neighborhood) on various aspects of children's health, including their oral health. As shown in Fig. 4, the percentage of parents who reported that their children had suboptimal oral health and other indicators of poor health rises with increases in the number of prevailing social risk factors, with the relationship between social risk factors and poor oral health demonstrating the steepest linear gradient. The proportion reporting less than very good teeth ranged from $14 \%$ for children with no social risk factors to $64 \%$ for children with $\geq 6$ social risk factors. Analyses that controlled for child age, gender, and number of children in the household showed an almost 11-fold increase in the odds for less than very good teeth in children with $\geq 6$ versus no social risks, underscoring the importance of social structure to healthy development.

Ecological Models of Caries and Periodontal Disease Figure 5 depicts a multidimensional
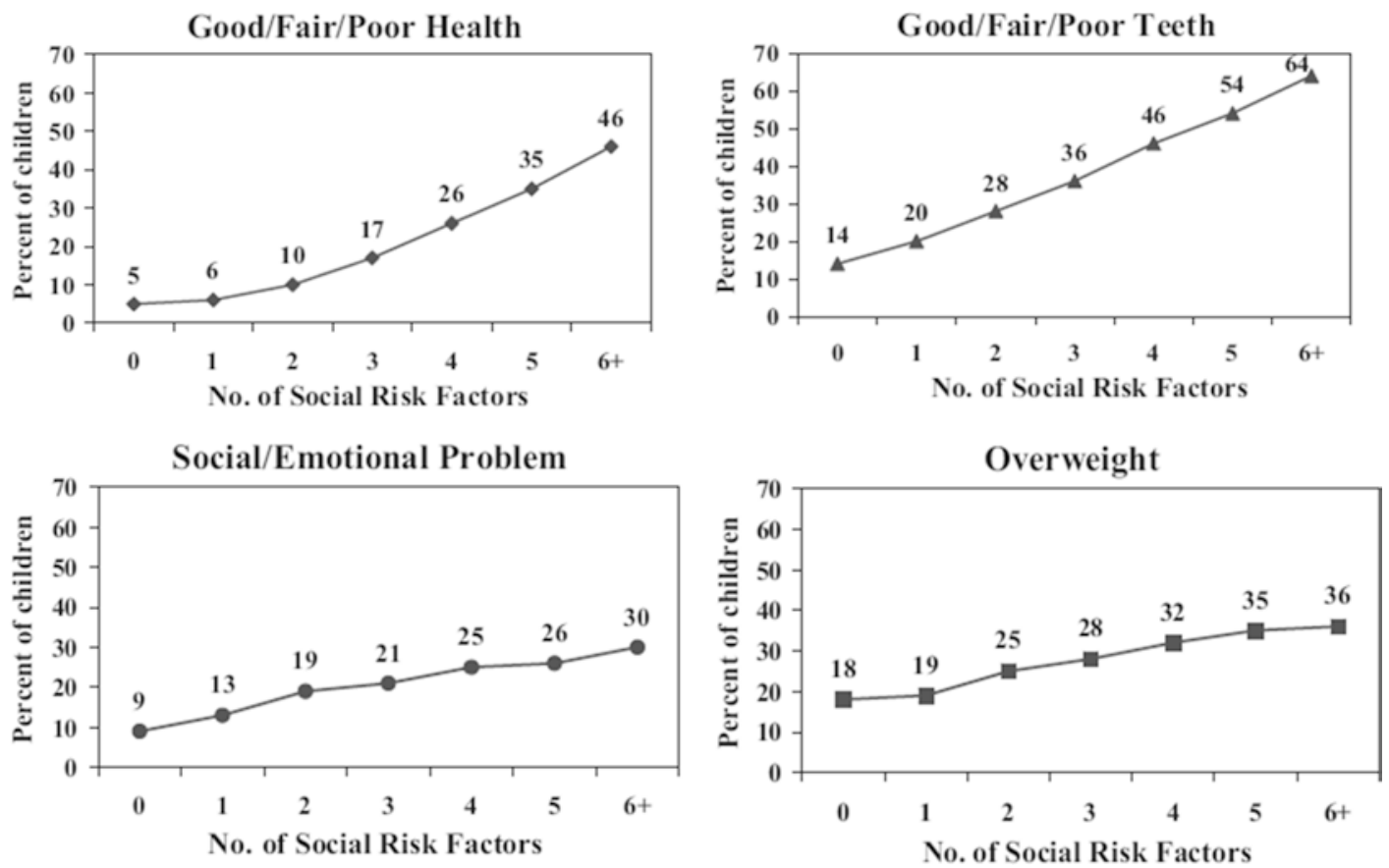

Fig. 4 Percentage of children in worse health by number of social risk factors (Source: Larson et al. 2008) 


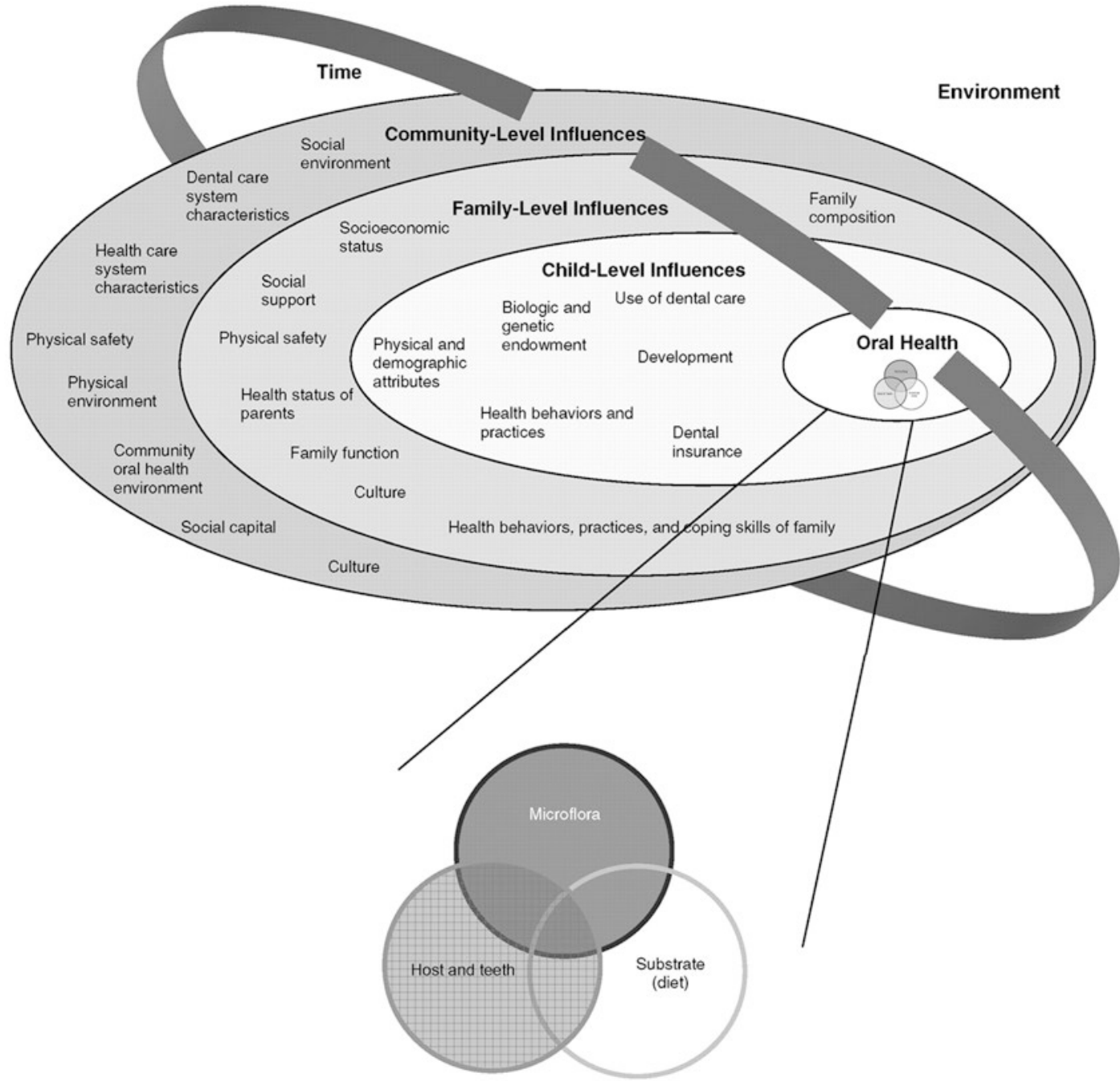

Fig. 5 Influences on children's oral health: a conceptual model (Source: Fisher-Owens et al. 2007)

ecological model for childhood dental caries, enumerating an array of biopsychosocial influences (including six child-level, eight familylevel, and eight community-level influences identified in the literature) in addition to the Keyes classic biological triad model and the influence of environmental factors and time (Fisher-Owens et al. 2007). The bulk of published studies underlying ecological models such as this have study design limitations similar to those identified in conducting the literature review for this chapter. Accordingly, their utility with respect to LCHD studies lies in helping to identify disease correlates and generate hypotheses, not in establishing oral disease or health development pathways.

Ecological models like the one shown in Fig. 5 have been used to design a variety of caries-risk assessment instruments. However, most cariesrisk assessment instruments include only a subset of the broad range of influences depicted in the Fisher-Owens model and generally have not undergone extensive validity testing (Quinonez and Crall 2009). 


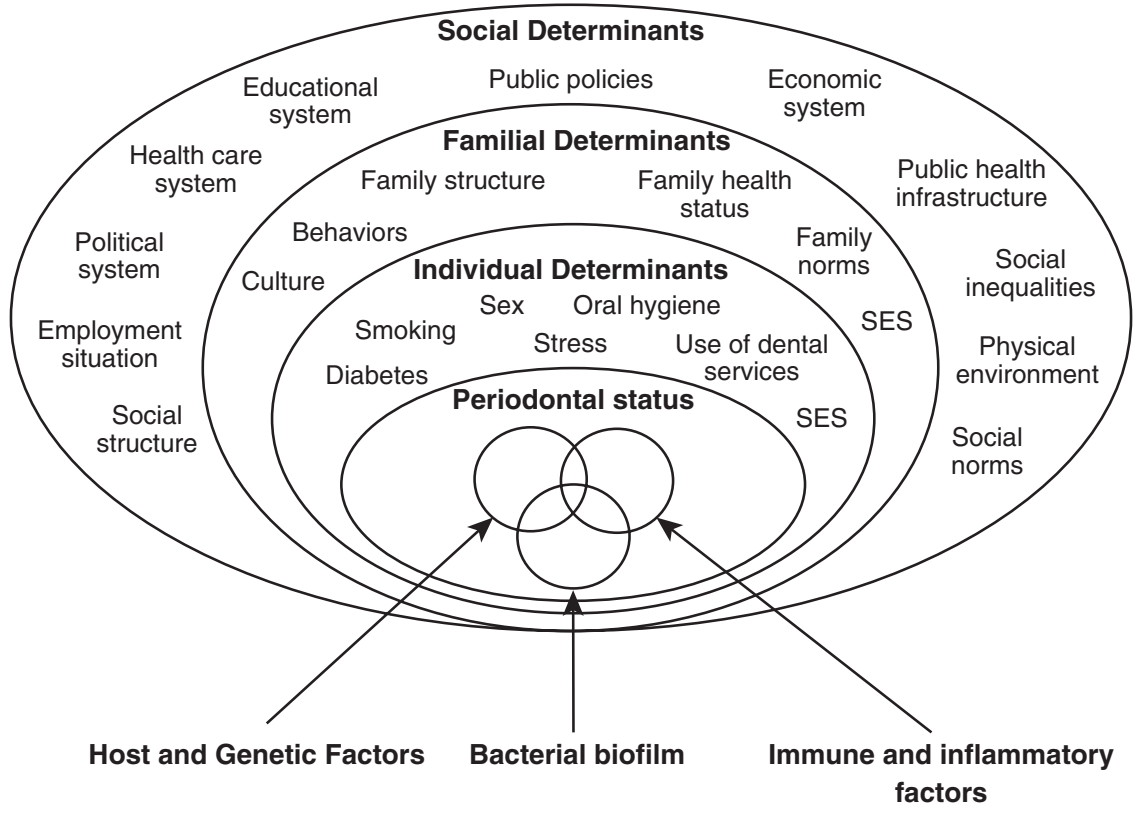

Fig. 6 Social determinants of oral health and disease in US men (Source: Tomar 2012)

Figure 6 depicts an ecological conceptual model for periodontal disease using a framework similar to the Fisher-Owens et al. model for childhood caries (Tomar 2012). In this case, the biological model emphasizes the interactions among host and genetic factors, bacteria associated with periodontal disease (different and distinct from cariogenic bacteria), and immune and inflammatory factors-reflecting a different pathogenic mechanism for periodontal disease than for dental caries. Constellations of individual, familial, and social influences are identified within categories of social, behavioral, and contextual factors in this model.

Ecological models like those shown in Figs. 5 and 6 are useful for depicting the complex array of etiological factors that potentially influence disease development, but are of limited value for explaining disease mechanisms or pathways as they do not explicitly incorporate developmental mechanisms or time into the frameworks. LCHD principles address these gaps by building on ecological models, adding time dimensions and developmental pathways to the models.

\section{Research Applications of Life Course Concepts to Oral Health}

\subsection{Life Course Epidemiology}

Life course epidemiology adds the time dimension to disease causation models, moving beyond the limitations of models such as those shown in the preceding section which depict an unspecified, essentially cross-sectional interplay among nested layers of environmental and individual factors. According to Kuh and Ben-Shlomo (Ben-Shlomo and Kuh 2002), "Life course epidemiology is defined as "the study of long-term effects on chronic disease risk of physical and social exposures during gestation, childhood, adolescence, young adulthood and later adult life. It seeks to understand causal links between exposures and outcomes, taking into consideration the importance of time (duration) and timing in the disease development." In a recent paper on concepts and theoretical models of life course epidemiology and its relevance to chronic oral 
conditions, Nicolau et al. (Nicolau et al. 2007) noted that "The life course approach to studying chronic disease etiology is not merely a collection of longitudinal data or the use of a particular study design or analytical method. Rather, the unique feature of this approach is a theoretical framework which assumes and tests a temporal ordering of exposure variables and their interrelationship with a specific outcome." The following section provides several examples of applications of life course epidemiology to studies of chronic oral diseases and conditions.

\subsection{Oral Health Trajectories: Dunedin Cohort Studies}

A number of studies published since the turn of the twenty-first century have begun to more fully embrace life course research methodologies and examine a broader array of life course influences. Foremost among them from the standpoint of assessing changes in oral health status over time and the influence of socioeconomic influences beginning in early childhood is the Dunedin Multidisciplinary Health and Development Study - a longitudinal investigation of health and behavior in a complete birth cohort of study members born in Dunedin, New Zealand, between April, 1972 and March, 1973. Findings of several Dunedin studies are summarized below.

Poulton et al. (Poulton et al. 2002) assessed a number of health outcomes, including dental caries, dental plaque scores, gingival bleeding, and periodontal disease status in a cohort of 1000 26-year-old Dunedin Study subjects whose socioeconomic status (SES) had been documented at birth and at ages, 3, 5, 7, 9, 11, 13, and 15 years. All dental health measures at age 26 years showed a graded relation with childhood SES. As SES increased, the amount of plaque and gingival bleeding and the proportion of individuals with periodontal disease and decayed surfaces decreased. The adverse influence of low childhood socioeconomic status was seen after controlling for infant health and contemporaneous adult SES. Additionally, the results showed that low adult SES had a significant effect on poor adult dental health after controlling for low childhood SES. The authors concluded that their "findings document that the social gradient in health-which has been amply documented among middle-aged and older adults - actually emerges in childhood. Whereas clinical and research interest in the social gradient has been generated mostly by studies of adults, the findings from this study suggest that the social gradient can be scrutinised in paediatric and adolescent populations as well. Further, whereas most studies of the social gradient have narrowed their attention to specific diseases, such as cardiovascular diseases, we document that the social gradient is far more ubiquitous and troubling. Low social class adversely affects many areas of people's health, including their physical, dental and mental health" (Poulton et al. 2002). This finding concerning children's dental health is highly noteworthy because there are few pediatric conditions that show a substantial SES gradient, which raises the possibility that body systems that develop rapidly and dramatically during childhood, such as the structures and physiological systems of the oral cavity, are more vulnerable to, and perhaps dependent on, environmental exposures.

Thomson et al. (2004) analyzed data on 789 Dunedin Study subjects at ages 5 and 26 years to investigate whether adult oral health status is influenced by (a) childhood socioeconomic advantage or disadvantage (controlling for childhood oral health) or (b) oral health in childhood (controlling for childhood socioeconomic advantage or disadvantage) and whether oral health in adulthood is affected by changes in SES. With respect to the question of whether poor adult oral health is predicted by socioeconomic disadvantage in childhood, after controlling for childhood oral health, analyses revealed that oral health inequalities present at age 5 years were also apparent at age 26 years when the early childhood SES categories were used, suggesting that early socioeconomic inequalities in a number of important oral health indicators do persist well into the third decade of life. Concerning the question of whether poor adult oral health is predicted 
by poor oral health in childhood, after controlling for childhood socioeconomic status, the evidence was unequivocal regarding dental caries: having high caries disease experience early in life predicted having greater disease experience in adulthood, other factors being equal. The pattern was not as clear with respect to periodontal disease, however. Finally, in examining the impact of individuals' SES at ages 5 and 26, Thomson and colleagues (Thomson et al. 2004) found that, for nearly all oral health indicators, a clear gradient for disease severity and prevalence was observed across socioeconomic trajectory groups in the following ascending order: "high (at age 5)-high (at age 26)," "low-high" (upwardly mobile), "high-low" (downwardly mobile), and "lowlow." These findings led the authors to conclude that adult oral health is predicted by not only childhood socioeconomic advantage or disadvantage but also by oral health in childhood. Changes in socioeconomic advantage or disadvantage between ages 5 and 26 were associated with differing levels of oral health in adulthood. Also of interest from the standpoint of LCHD is the possibility that oral health may represent a sentinel system, with early manifestations of disease serving as a harbinger for elevated risk of other chronic diseases (e.g., obesity, diabetes).

Broadbent et al. (2008) investigated longitudinal patterns of caries experience in a birth cohort of 955 Dunedin Study subjects in order to identify and describe developmental trajectories of caries experience in the permanent dentition using data collected from dental examinations at ages $5,9,15,18,26$, and 32 years. Three caries experience trajectory classes were identified: "high" (15\% of subjects), "medium" (43\%), and "low" (42\%) with respect to decayed, missing, and filled (tooth) surfaces (DMFS). All trajectories were relatively linear, although the higher trajectories were more "S-shaped" (Fig. 7). This effect disappeared following adjustment for the number of unaffected surfaces remaining at each age, suggesting that, among individuals following a similar caries trajectory, the caries rate is relatively constant across time. This finding is

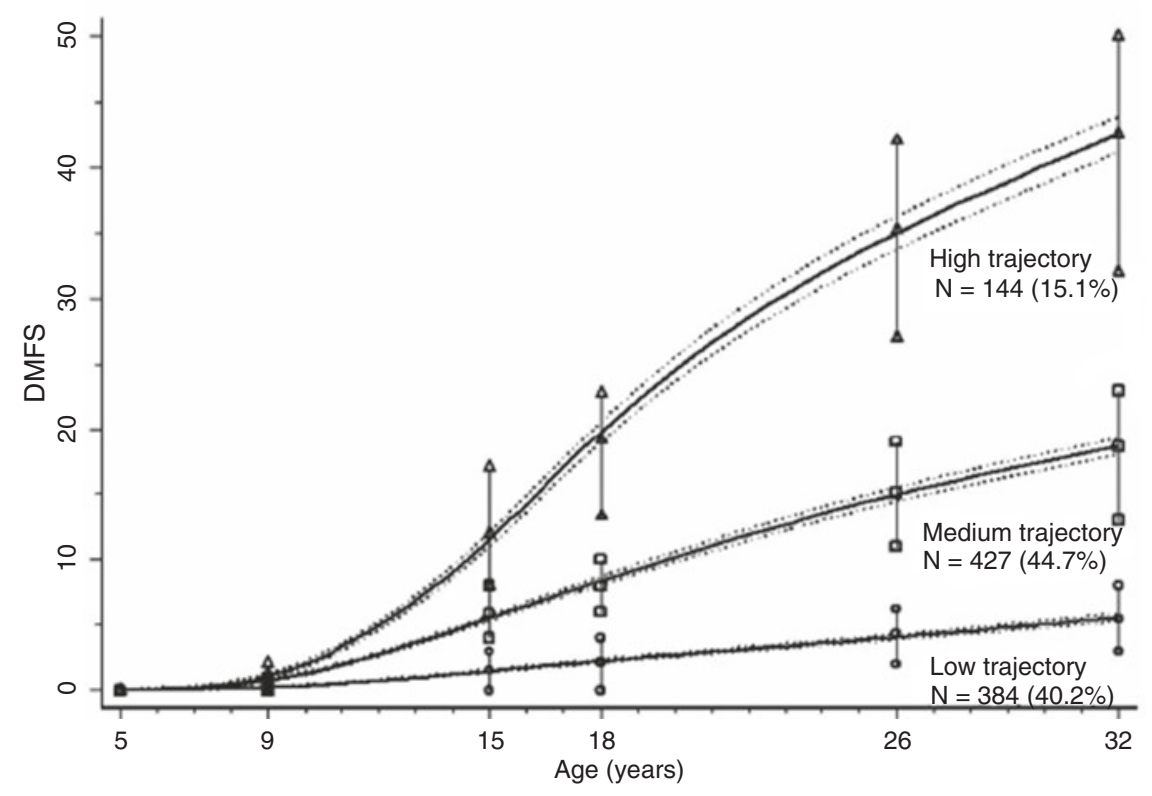

Bars represent inter quartile range, dotted lines represent $95 \% \mathrm{Cl}$ DMFS: Decayed, Missing, and Filled surfaces

Fig. 7 Trajectory patterns of dental caries experience in the permanent dentition to the fourth decade of life (Source: Broadbent et al. 2008) 
consistent with observations by Fejerskov (2004) that the caries incidence rate in a group of individuals appears fairly constant throughout life if no special efforts to control lesion progression are made. Broadbent et al. (2008) concluded that these results did not support the commonly held belief among dentists that childhood and adolescence are periods of special risk for dental caries or that caries "immunity" may be acquired during late adolescence or early adulthood. In fact, other studies have reported that ECC is one of the most reliable predictors of elevated risk of caries experience later in life.

Crocombe et al. (2011) also analyzed data from 833 participants in the Dunedin Study to assess the impact of dental visiting trajectory patterns on clinical oral health and oral healthrelated quality of life measures. Information on the use of dental services was collected at ages 15, 18, 26, and 32. Dental visiting trajectories were significantly associated with both measures of self-reported oral health and three of the four dental clinical indicators. The regular dental attendance group had less than half the mean number of missing teeth, a lower mean DMFS score, and a lower mean decayed surfaces (DS) than those defined as "opportunists" and "decliners." The regular group had the lowest mean Oral Health Impact Profile (OHIP-14) score and was nearly twice as likely as opportunists and decliners to report that they had better-than-average oral health. Low SES and being dentally anxious were associated with worse health outcomes for all outcome variables, while "poor plaque trajectory" and smoking were associated with worse outcomes for all variables except filled surfaces (FS). The influence of dental visiting trajectory on clinical oral health outcomes was much higher at lower SES levels than higher SES levels, leading the authors to conclude that improving dental visiting behavior among people of lower SES would improve clinical oral health outcomes more and have a greater reduction of oral health impacts than it would for people of higher SES.

A number of additional studies of shorter duration and/or employing retrospective meth- ods of data collection (which may be subject to some degree of recall bias) have been conducted in other countries, most notably Brazil, Norway, the United Kingdom, and Hong Kong (Lu et al. 2011; Holst; Peres et al. 2011; Mason et al. 2006). Collectively, these studies generally affirm the findings from analyses conducted as part of the Dunedin Study, with some variation that likely reflects varying levels of SES (particularly poverty) and differences in the types of oral health-care systems and programs available across countries. Many of these studies are limited to adolescents (starting at age 15) or adults. An exception is the work of (Holst and Schuller 2011) which examined different birth cohorts in Norway and found that the health environment in childhood was important for adults' oral health during most of the twentieth century. Attention from parents and the local environment lead to better oral health outcomes in adulthood. Social status measured by length of education also was associated with choices leading to better oral health. Regular dental visits were important especially for the eldest birth cohort. Good oral health behaviors early and during adulthood were important for oral health.

The studies summarized in this section represent advances in our understanding of the impacts of various influences on oral health over the life course. Interest in using a life course perspective to study various aspects of oral health has grown steadily in recent times. Main lines of pursuit include investigations of critical periods and accumulation of risk models, which have shed light on the importance of early life experiences, oral health trajectories, and the importance of social determinants to oral and general health (Heilman et al. 2015). However, it is worth noting that life course epidemiology only reflects the principles of LCHD (see Table 1) in a very limited way, primarily with respect to LCHD Principle 3 (Complexity: multidimensional person-environmental interactions) and LCHD Principle 5 (Timing: health is highly sensitive to timing and social structuring of environmental exposures). 


\subsection{Relating Oral Health, General Health, and Quality of Life}

Summarizing what is known about the influences of oral health and oral health problems on life course outcomes, general health and overall quality of life is a daunting challenge that goes beyond the scope of this chapter. In lieu of attempting such a synopsis, the reader is referred initially to the US Surgeon General's Report on Oral Health (US Department of Health and Human Services 2000) for a broad introduction to the literature. Chapters 5 and 6 of that extensively researched and widely vetted document are summarized below.

Chapter 5 of the SGROH examines multiple linkages between oral and general health, noting that the mouth and the face reflect signs and symptoms of health and disease that can serve as an adjunct for diagnosis for some conditions. Diagnostic tests using oral cells and fluidsespecially saliva - are available to detect drug abuse, hormonal changes, and specific diseases, and more are being developed. The mouth also is a portal of entry for pathogens and toxins, which can affect the mouth and, if not cleared by the many defense mechanisms that have evolved to protect the oral cavity, may spread to the rest of the body. Recent epidemiologic and experimental animal research provides evidence of possible associations between oral infections-particularly periodontal disease - and diabetes, cardiovascular disease, and adverse pregnancy outcomes. The review in Chapter 5 of the SGROH also highlights the need for an aggressive research agenda to better delineate the specific nature of these associations and the underlying mechanisms of action.

Chapter 6 of the SGROH looks at the impact of oral health problems on quality of life and includes examples of the kinds of questionnaires used to measure oral-health-related quality of life. Oral health generally is highly valued by society and individuals, and the chapter begins with a brief description of the reflections of those values in myth and folklore concerning facial appearance and the meaning of teeth. It then explores dimensions beyond the biological and the physical to examine how oral diseases and disorders can interfere with the functions of daily living, including participation in work or school, and what is known about their psychosocial impacts and economic costs. The deleterious effects of facial disfigurement and tooth loss may be magnified in modern societies that celebrate youth and beauty. Self-reported impacts of oral conditions on social functions include limitations in communication, social interactions, and intimacy. Additional research on oral-health-related quality of life is recommended to permit further exploration of the dimensions of oral health and well-being.

Sheiham (2005) also has provided a commentary on the relationships among oral health, general health, and quality of life. Many of the points highlighted in this commentary relate to LCHD Principle 1 (Health Development) that health is an emergent property and that oral health is a component of and contributes to overall health. Sheiham's summary ultimately reiterates the SGROH's call for cessation of "the compartmentalization involved in viewing the mouth separately from the rest of the body, noting that oral health affects general health by causing considerable pain and suffering and by altering what people eat, their speech, and their quality of life and well-being."

Additional excerpts from Sheiham's commentary include the following. "Oral health also has an effect on other chronic diseases, and failure to tackle social and material determinants and incorporate oral health into general health promotion means that millions suffer intractable toothache and poor quality of life, and end up with few teeth.

Oral diseases are the most common of the chronic diseases and are important public health problems because of their prevalence, their impact on individuals and society, and the expense of their treatment. The general determinants of oral diseases are known and include risk factors common to a number of chronic diseases: diet, inadequate hygiene practices, smoking, alcohol, risky behaviors causing injuries, and stress. Moreover, effective methods are available to prevent or reduce the impact of major oral diseases. 
Oral health affects people physically and psychologically and influences how they grow, enjoy life, look, speak, chew, taste food and socialize, as well as their feelings of social well-being. Severe caries detracts from children's quality of life: they experience pain, discomfort, disfigurement, acute and chronic infections, and eating and sleep disruption as well as higher risk of hospitalization, high treatment costs and loss of school days with the consequently diminished ability to learn. Caries affects nutrition, growth and weight gain. Children of three years of age with nursing caries weigh about $1 \mathrm{~kg}$ less than control children because toothache and infection alter eating and sleeping habits, dietary intake and metabolic processes. Disturbed sleep affects glucocorticoid production. In addition, there is suppression of hemoglobin from depressed erythrocyte production.

Chronic diseases such as obesity, diabetes and caries are increasing in developed and developing countries, with the implication that quality of life related to oral health, as well as general quality of life, may deteriorate. Because oral and other chronic diseases have determinants in common, more emphasis should be on the common risk factor approach. The key concept underlying future oral health strategies is integration with this approach, a major benefit being the focus on improving health conditions in general for the whole population and for groups at high risk, thereby reducing social inequities."

\section{$5 \quad$ Relating Oral Health and LCHD Concepts}

\subsection{Relating Oral Health Research Findings to Key LCHD Concepts}

Pathways and Trajectories Analyses based on the Dunedin prospective, longitudinal birth cohort studies have distinguished three oral health trajectory groupings (high, medium, low) that emerge as early as age 5 , remain relatively constant over time, and exhibit increasing divergence through the third decade of life. Authors of the Dunedin Study acknowledge that aggregating their findings into three trajectory 'groups' is an analytical convenience that may mask the heterogeneity of individuals within each group. To that point, LCHD suggests that a focus on intraindividual trajectories rather than on "group" means may prove more insightful because it allows for careful ascertainment of temporal exposure-outcome relationships and allows the analysis to take advantage of the diversity and complexity of these interactions rather than masking variability by aggregation into a few classes. Classes assume homogeneity of persons within a group, an assumption that is generally not supported. Little if any investigations of intraindividual change with detailed ascertainment of exposure and outcome over time have been reported in the dental/ oral health literature.

Additional Dunedin cohort studies have examined the effect of SES in childhood and adulthood on adult oral health, and the effect of early childhood oral health on adult oral health, and found that the highest oral health trajectories are exhibited by those who have higher SES and better oral health in childhood; the lowest trajectories are found in those who have low SES and poor oral health in childhood; and those whose SES changed (either upwardly or downwardly) between childhood and adulthood fall between the other two trajectories. Oral health-care utilization and other personal, family, and community factors (e.g., oral hygiene, diet, dental anxiety, emphasis placed on oral health within one's family or community) also appear to exert influences on oral health trajectories throughout the life course.

Early Programming Evidence concerning the impact of early programming on oral health over the lifespan is less well defined, in part because many studies focus on adolescents or adults. Developmental disturbances that result in structural anomalies of tooth structure represent one documented example of early programming influences that can increase risk for dental disease (Targino et al. 2011). Findings from studies conducted by Holst and Schuller (2011) and Alm (Alm et al. 2008) suggest that good oral hygiene 
habits, including the use of fluoride toothpaste, established in early childhood provide a foundation for a low risk of proximal caries in adolescents. Evidence for the influence of less direct influences such as low birth weight is more equivocal, but represents an area of growing research interest (Nicolau et al. 2007). Evidence of intergenerational programming has been documented, particularly with respect to maternalchild oral health relationships (Shearer and Thomson, 2010; Shearer et al. 2011).

Critical or Sensitive Periods Some evidence, including studies cited above, supports the critical or sensitive period hypothesis with respect to the impact of various influences during fetal, early childhood, and later stages on life course oral health development and the determinants of oral health disparities. The importance and etiological mechanisms of disturbances arising during the prenatal period on craniofacial developmental problems such as cleft lip and/or cleft palate have been extensively documented. However, much remains to be elucidated with respect to the effects of different influences, at different times, on other oral health-related conditions (dental caries, periodontal disease, cancer, TMJD) (Hallqvist et al. 2004).

Cumulative Impact Substantial evidence also exists to support the hypothesis that the impact of major oral health-related conditions is a function of cumulative experiences or episodes of disease over time. Clearly, it is well established that dental caries is a chronic, cumulative disease and that the caries status of an individual develops and is subject to biological, behavioral, social, and environmental influences over time. Caries occurs at any stage in life, provided that an individual has susceptible teeth (and surfaces) remaining (Broadbent et al. 2008). The same case can be made for periodontal disease and tooth loss resulting from caries and periodontal diseases (Shearer et al. 2011; Watt 2007).

Risk and Protective Factors Considerable evidence has been compiled with respect to the biological and, to a lesser extent, behavioral risk and protective factors for major dental conditions (see Figs. 5 and 6). The influence of genetic, epigenetic, family, community, social, and environmental risk factors, in individual populations or across populations, is less well documented and understood.

\subsection{Gaps in Knowledge Concerning Oral Health from a LCHD Perspective}

Researchers involved in studying life course influences on oral health have identified a number of gaps in the current knowledge base. Using the lens of the LCHD principles, we have compiled research needs for future studies on oral health:

- Evaluate the contribution of social isolation, social relationships, and social support to oral health.

- Obtain more information on the economic, political, social, and environmental causes of individual behaviors related to oral health.

- Increase the number of longitudinal studies assessing the long-term effects of early childhood caries (ECC) and treatment on the health and quality of life of preschool children.

- Longitudinal studies are needed in order to obtain more knowledge about causative factors and the possible relationships between dental caries and overweight/obesity in children.

- Conceptual work that articulates a definition of oral health motivated by the LCHD principles is needed.

- Theoretical explanations for health inequalities are limited and biased due to the types of data collected in modern epidemiological studies, which are geared toward identifying and quantifying risk factors for disease and intended as a basis for description (of disease), not explanation (of causal pathways). This raises questions about the validity of current explanations. Current epidemiological approaches are widely criticized for neglecting broad social factors and failing to dig below the surface into issues such as how differ- 
ent social class groups live their lives and what factors influence their lifestyle decisions. Current explanations are based largely on what epidemiological researchers can see and measure; factors that are harder to assess (such as culture), but which may be critically important to advancing our understanding of social inequalities in health, are frequently neglected.

- Epidemiologists have largely persisted in the use of disease-based measures to assess inequalities in health and oral health. The most significant advance to our understanding of social inequalities in health may come from the creation of a data set that measures oral health as defined by the FDI or oral-healthrelated quality of life.

- To date there is no research exploring how members of the population understand and account for inequalities in oral health, which remains a significant omission as it could offer promising new insights.

- Further research is needed to clarify the apparently differing oral health beliefs, attitudes, and practices of lower and higher SES groups.

- Much work remains to be done to establish which biological, social, and environmental factors are determinants of oral health or disease and which are merely markers, "proxies," or confounders.

- The process by which social stratification translates to poor oral health beginning in earliest childhood, especially for groups at higher risk for disease, is not well understood.

- There is a need for future research to move beyond traditional risk factors and more closely examine the impact of the social environment on oral health beliefs, behavior, and outcomes.

- A number of oral health problems have been shown to be associated with other health conditions (e.g., low birth weight, cardiovascular disease, respiratory disease); however, the demonstration of actual causal connections or pathways, such as the bidirectional relationship between diabetes and periodontal disease, has been modest.

\subsection{Recommendations for Research and Policy Priorities}

The knowledge gaps noted above give rise to a number of recommendations for research priorities to better understand oral health life course influences and policy changes to help reduce the impact of oral health problems and reduce oral health inequalities, which can be summarized as follows:

- Greater support for longer-term longitudinal oral health life course studies with detailed and frequent data collection to allow for the construction of intraindividual trajectories

- Replication of true oral health life course studies in different populations to assess whether findings apply across diverse settings

- Greater emphasis on research delineating relationships among oral health determinants to identify causal pathways

- Greater emphasis on identifying and addressing broader influences on oral health (i.e., family, community, social, and environmental influences)

\section{Summary}

This chapter has outlined major concepts embodied in the Life Course Health Development framework, examined evidence relating various aspects of major oral health-related conditions to this framework, and produced recommendations for advancing research and policy concerning oral health. LCHD provides a highly useful approach for understanding oral health determinants, disparities, and influences on general health and well-being and for advancing knowledge, policies, and programs to optimize health across individuals and populations.

\section{References}

Agency for Healthcare Research and Quality. 2015. Dental Services Mean and Median Expenses per Person With Expense and Distribution of Expenses by Source of Payment: United States, 2012. Medical Expenditure 
Panel Survey Household Component Data. Generated interactively. (3 Mar 2015).

Alm, A., et al. (2008). Oral hygiene and parent-related factors during early childhood in relation to approximal caries at 15 years of age. Caries Research, 42(1), 28-36.

American Cancer Society. (2015). Cancer Facts \& Figures 2015. Atlanta: American Cancer Society.

Ben-Shlomo, Y., \& Kuh, D. (2002). A life course approach to chronic disease epidemiology: Conceptual models, empirical challenges and interdisciplinary perspectives. International Journal of Epidemiology, 31, 285-293.

Berkowitz, R. J. (2006). Mutans steptococci: Acquistion and transmission. Pediatric Dentistry, 28, 106-109.

Borrell-Carrio, F., Suchman, A. L., \& Epstein, R. M. (2004). The biopsychosocial model 25 years later: Principles, practice, and scientific inquiry. Annals of Family Medicine, 2, 576-582.

Boulet, S. L., Grosse, S. D., Honein, M. A., \& CorreaVillasenor, A. (2009). Children with orofacial clefts: Health care use and costs among a privately insured population. Publ Health Reports, 124, 447-453.

Brennan, D. S., Spencer, A. J., \& Roberts-Thomson, K. F. (2008). Tooth loss, chewing ability and quality of life. Quality of Life Research, 17, 227-235.

Broadbent, J. M., Thomson, W. M., \& Poulton, R. (2008). Trajectory patterns of dental caries experience in the permanent dentition to the fourth decade of life. Journal of Dental Research, 87, 69-72.

Center for Disease Control and Prevention (CDC). (2014). Prevalence (number of cases) of cleft lip and cleft palate. Available at: http://www. nidcr.nih.gov/DataStatistics/FindDataByTopic/ CraniofacialBirthDefects/PrevalenceCleft $\% 20$ LipCleftPalate.htm.

Crocombe, L. A., et al. (2011). Dental visiting trajectory patterns and their antecedents. Journal of Public Health Dentistry, 71(1), 23-31.

Dye, B. A., Thornton-Evans, G., Li, X., \& Iafolla, T. J. (2015a). Dental caries and sealant prevalence in children and adolescents in the United States, 2011a2012. NCHS data brief, no 191. Hyattsville: National Center for Health Statistics.

Dye, B. A., Thornton-Evans, G., Li, X., \& Iafolla, T. J. (2015b). Dental caries and tooth loss in adults in the United States, 2011b-2012. NCHS data brief, no 197. Hyattsville: National Center for Health Statistics.

Feathersone, J. B. D. (2000). The science and practice of caries prevention. Journal of the American Dental Association (Chicago, IL), 131, 887-899.

Fejerskov, O. (2004). Changing paradigms in concepts on dental caries: Consequences for oral health care. Caries Research, 38, 182-191.

Fine, A., \& Kotelchuck, M. (2010). Rethinking MCH: The life course model as an organizing framework. Washington, DC: US Department of Health and Human Services, Health Resources and Services Administration, Maternal and Child Health Bureau.

Fisher-Owens, S. A., Gansky, S. A., Platt, L. J., et al. (2007). Influences on children's oral health: A conceptual model. Pediatrics, 120, e510-e520.
Fitzgerald, R. J., \& Keyes, P. H. (1961). Demonstration of the etiologic role of streptococci in experimental caries in the hamster. Journal of the American Dental Association (Chicago, IL), 61, 9-19.

Gerabek, W. E. (1999). The tooth-worm: Historical aspects of a popular medical belief. Clin Oral Invest, 3, 1-6.

Glick, M., et al. (2016). A new definition for oral health developed by the FDI World dental federation opens the door to a universal definition of oral health. Journal of the American Dental Association (Chicago, IL), 147, 915-917.

Gregory, J., Gibson, B., \& Robinson, P. G. (2005). Variation and change in the meaning of oral health related quality of life: A 'grounded' systems approach. Social Science \& Medicine, 60, 1859-1868.

Halfon, N., \& Hochstein, M. (2002). Life course health development: An integrated framework for developing health, policy, and research. The Milbank Quarterly, 80, 433-479.

Halfon, N., Larson, K., Lu, M., Tullis, E., \& Russ, S. (2014). Lifecourse health development: Past, present and future. Maternal and Child Health Journal, 18, 344-365.

Hallqvist, J., et al. (2004). Can we disentangle life course processes of accumulation, critical period and social mobility? An analysis of disadvantaged socioeconomic positions and myocardial infarction in the Stockholm heart epidemiology program. Social Science \& Medicine, 58(8), 1555-1562.

Heilman, A., et al. (2015). Oral health over the life course. In C. Burton-Jeangros, S. Cullati, \& A. Sacker (Eds.), A life course perspective on health trajectories and transitions [Internet]. Cham $(\mathrm{CH})$ : Springer. doi:10.1007/9783319204840_3.

Hollister, M. C., \& Weintraub, J. A. (1993). The association of oral status with systemic health, quality of life, and economic productivity. Journal of Dental Education, 57(12), 901-912.

Holst, D., \& Schuller, A. A. (2011). Equality in adults' oral health in Norway. Cohort and cross-sectional results over 33 years. Community Dent Oral Epidemiol, 39(6), 488-497. doi:10.1111/j.1600-0528.2011.00624.x.

Jacobson, J. J., et al. (2012). The cost burden of oral, oral pharyngeal, and salivary gland cancers in three groups: Commercial insurance, Medicare, and Medicaid. Head \& Neck Oncology, 4, 15.

Keating, D. P., \& Hertzman, C. (1999). Developmental health and the wealth of nations : Social, biological, and educational dynamics. New York, NY: Guilford Press.

Keyes, P. H. (1960). The infectious and transmissible nature of experimental dental caries. Archives of Oral Biology, 1, 304-320.

Kohli, S. S., \& Kohli, V. S. (2012). A comprehensive review of the genetic basis of cleft lip and palate. J Oral Maxillofac Pathol., 16, 64-72. doi:10.4103/0973-029X.92976.

Larson, K., Russ, S. A., Crall, J. J., \& Halfon, N. (2008). Influence of multiple social risks on children's health. Pediatrics, 121, 337-344. 
Liu, Z., et al. (2009). The impact of malocclusion/orthodontic treatment need on the quality of life: A systematic review. The Angle Orthodontist, 79, 585-591.

Locker, D., \& Slade, G. (1994). Association between clinical and subjective indicators of oral health status in an older adult population. Gerodontology, 11, 108-114.

Lu, H. X., et al. (2011). Trends in oral health from childhood to early adulthood: A life course approach. Community Dentistry and Oral Epidemiology, 39(4), $352-360$.

Mason, J., et al. (2006). How do factors at different stages of the lifecourse contribute to oral-health-related quality of life in middle age for men and women? Journal of Dental Research, 85(3), 257-261.

Miller, W. D. (1890). The micro-organisms of the human mouth. Philadelphia: S.S. White Publishing Co..

Miller, W. D. (1891). The human mouth as a focus of infection. Dental Cosmos, 33, 689-706.

Nicolau, B., et al. (2007). Life-course epidemiology: Concepts and theoretical models and its relevance to chronic oral conditions. Community Dentistry and Oral Epidemiology, 35(4), 241-249.

NIDCR. (2003). Prevalence of TMJD and its signs and symptoms; Available from: http://www.nidcr.nih. gov/DataStatistics/FindDataByTopic/FacialPain/ PrevalenceTMJD.htm.

Peres, M. A., et al. (2011). The influence of family income trajectories from birth to adulthood on adult oral health: Findings from the 1982 Pelotas birth cohort. American Journal of Public Health, 101(4), 730-736.

Poulton, R., et al. (2002). Association between children's experience of socioeconomic disadvantage and adult health: A life-course study. Lancet, 360(9346), 1640-1645.

Quinonez, R., \& Crall, J. J. (2009). Caries-risk assessment in early childhood. In J. H. Berg \& R. Slayton (Eds.), Early childhood oral health. Hoboken: Wiley-Blackwell.

Ram, H., Kumar, H., Konwar, R., Bhatt, M. L. B., \& Mohammad, S. (2011). Oral cancer: Risk factors and molecular pathogenesis. J Maxillofac Oral Surg, 10, 132-137.

Shearer, D. M., \& Thomson, W. M. (2010). Intergenerational continuity in oral health: A review. Community Dentistry and Oral Epidemiology, 38(6), 479-486.

Shearer, D. M., et al. (2011). Maternal oral health predicts their children's caries experience in adulthood. Journal of Dental Research, 90(5), 672-677.
Sheiham, A. (2005). Oral health, general health and quality of life. Bulletin of the World Health Organization, 83(9), 644-644.

Sheiham, A., \& Watt, R. G. (2000). The common risk factor approach: A rational basis for promoting oral health. Community Dentistry and Oral Epidemiology, 28, 399-406.

Targino, A. G. R., et al. (2011). The relationship of enamel defects and caries: A cohort study. Oral Diseases, 17(4), 420-426.

The Oral Cancer Foundation. (2015). Oral cancer facts. Available at: http://www.oralcancer.org/facts/.

Thomson, W. M., et al. (2004). Socioeconomic inequalities in oral health in childhood and adulthood in a birth cohort. Community Dentistry and Oral Epidemiology, 32, 345-353.

Thornton-Evans, et al. (2013). Periodontitis among adults aged > United States, 2009-2010. MMWR, 62(3), $129-135$.

Tomar, S. L. (2012). Social determinants of oral health and disease in US men. Journal of Mens Health, 9, 113-119.

U.S. Department of Health and Human Services. (2000). Oral Health in America: A Report of the Surgeon General. Rockville: U.S. Department of Health and Human Services, National Institute of Dental and Craniofacial Research, National Institutes of Health.

Vargas, C. M., Crall, J. J., \& Schneider, D. A. (1998). Sociodemographic distribution of pediatric dental caries: NHANES III, 1988-1994. Journal of the American Dental Association (1939), 129(9), 1229-1238.

Wall, T., \& Vujicic M. (2014). No growth in U.S. dental spending in 2013. Health policy Institute research brief. American Dental Association. December 2014. Available from: http://www.ada.org/ /media/ ADA/Science \%20 and\%20Research/HPI/Files/ HPIBrief_1214_4.ashx.

Watt, R. G. (2007). From victim blaming to upstream action: Tackling the social determinants of oral health inequalities. Community Dentistry and Oral Epidemiology, 35(1), 1-11.

WHO, The World Oral Health Report 2003. (2008). Continuous improvement of oral health in the $21 \mathrm{st}$ century-the approach of the WHO global oral health Programme. Community Dent Oral Epidemiol, 31(s1), $3-24$.

Open Access This chapter is licensed under the terms of the Creative Commons Attribution 4.0 International License (http://creativecommons.org/licenses/by/4.0/), which permits use, sharing, adaptation, distribution and reproduction in any medium or format, as long as you give appropriate credit to the original author(s) and the source, provide a link to the Creative Commons license and indicate if changes were made.

The images or other third party material in this chapter are included in the chapter's Creative Commons license, unless indicated otherwise in a credit line to the material. If material is not included in the chapter's Creative Commons license and your intended use is not permitted by statutory regulation or exceeds the permitted use, you will need to obtain permission directly from the copyright holder.

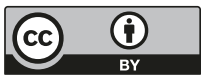

